

\title{
Medium modifications of kaons in pion matter
}

\author{
B.V. Martemyanov ${ }^{a, b)}$, Amand Faessler ${ }^{b)}$, C. Fuchs $^{b)}$ and M.I. Krivoruchenko ${ }^{a, b)}$ \\ ${ }^{a)}$ Institute for Theoretical and Experimental Physics, B. Cheremushkinskaya 25 \\ 117259 Moscow, Russia \\ ${ }^{b)}$ Institut für Theoretische Physik, Universität Tübingen, Auf der Morgenstelle 14 \\ D-72076 Tübingen, Germany
}

Kaon in-medium masses and mean-field potentials are calculated in isotopically symmetric pion matter to one loop of chiral perturbation theory. The results are extended to RHIC temperatures using experimental data on $\pi K$ scattering phase shifts. The kaon in-medium broadening results in an acceleration of the $\phi \rightarrow K \bar{K}$ decay. The increased apparent dilepton branching of the $\phi$-mesons, observed recently by NA50, NA49, and the PHENIX collaborations at RHIC, is interpreted in terms of rescattering of secondary kaons inside of the pion matter.

keywords: kaons, pions, heavy-ion collisions

The theoretical study of kaon properties in a dense nuclear environment has a long history [1-3]. To search for signals of predicted in-medium modifications at high baryon densities is one of the primary goals of the experiments devoted to the measurements of kaonic observables in heavy-ion reactions at intermediate energies [4]. Corresponding transport calculations revealed significant evidence for in-medium modifications of the kaon properties during the course of such reactions $[5,6]$. This picture was recently complemented by the measurements of $K^{+}$ production in proton-nucleus reactions [7].

In heavy-ion reactions at RHIC energies, the physical conditions are quite different. The medium is baryon dilute but meson rich [8]. Since by far the most abundant particles are pions, one can speak about pion matter created in heavy-ion collisions at RHIC energies. It is therefore interesting to consider the modifications of kaon properties in such a pion-dominated medium. This question is also important from another point of view: The $\phi$-meson is considered as a promising mesonic probe for the fireball formed in such reactions $[9,10]$.

Possible modifications of the $\phi$-meson dilepton branching in a pion gas have been discussed one decade ago by Lissauer and Shuryak [11] and by Blaizot and Galain [12] . The $\mu^{+} \mu^{-}$yield from $\phi$-meson decays was recently measured in central $\mathrm{Pb}+\mathrm{Pb}$ collisions at CERN/SPS energies by the NA50 Collaboration [13] and the $K \bar{K}$ yield was measured by the NA49 Collaboration [14]. The number of $\phi$-mesons detected through the dilepton channel was found to be a factor of two to four greater than that of $\phi$-mesons detected trough the kaon channel. This difference might be attributed to in-medium $\phi$ and $K$ mass shifts [12] and/or rescattering of the secondary kaons in hadronic matter $[15,16]$. Recently, preliminary $\phi$ - meson production data in $\mathrm{Au}+\mathrm{Au}$ collisions at $\mathrm{RHIC}$ energies were reported by the PHENIX Collaboration [17]. In this experiment, the $\phi$ yield was simultaneously measured in the $\phi \rightarrow e^{+} e^{-}$and $K \bar{K}$ channels. The result is consistent with an increased dilepton branching.

The kaon-pion gas was studied in details within chiral perturbation theory (ChPT) in Refs. [18,19], focusing thereby on the quark condensates. In this letter, we discuss modifications of the kaon self-energy operator $\Sigma\left(p^{2}, E\right)$ and of the $\phi \rightarrow K \bar{K}$ decay mode inside hot pion matter. ChPT, proposed for description of interactions of pseudoscalar mesons at low energies, is an adequate tool for studying the problem at low temperatures and useful to control the low temperature limit of phenomenological models.

The in-medium mass operator of kaons, $\Sigma\left(p^{2}, E\right)$, can be expressed in terms of the $\pi K$ forward scattering amplitudes for on-shell pions and off-shell kaons. The onshell $\pi K$ amplitudes have been calculated in ChPT to the order $p^{4}$ by several authors (see e.g. [20] and references therein). To lowest order $p^{2}$, the off-shell $\pi K$ amplitudes are given in Ref. [21]. Near the threshold, the isospineven and odd $\pi K$ scattering amplitudes can be written as

$$
\begin{aligned}
A^{ \pm}\left(s, t, p^{2}\right) & =8 \pi \sqrt{s}\left(a_{0}^{ \pm}+p^{* 2}\left(b_{0}^{ \pm}+3 a_{1}^{ \pm}\right)+\frac{3}{2} t a_{1}^{ \pm}\right) \\
& +c^{ \pm}\left(p^{2}-M_{K}^{2}\right)
\end{aligned}
$$

where $a_{\ell}^{ \pm}$and $b_{\ell}^{ \pm}$are the $\pi K$ scattering lengths and effective ranges, $p^{*}=p^{*}\left(\sqrt{s}, M_{\pi}, M_{K}\right)$ is the c.m. momentum of the $\pi K$ system, $s=\left(p+p_{\pi}\right)^{2}, t=\left(p_{\pi}^{\prime}-p_{\pi}\right)^{2}, p=(E, \mathbf{p})$ is the kaon momentum and $p^{2} \neq M_{K}^{2}$ in general.

The number densities of pions are given by Bose distributions

$$
d n_{v \pi}=\frac{d^{3} p_{\pi}}{(2 \pi)^{3}}\left(\exp \left(\frac{E_{\pi}-\mu_{\pi}}{T}\right)-1\right)^{-1}
$$

where $\mu_{\pi^{+}}=-\mu_{\pi^{-}}$is the $\pi^{+}$chemical potential, $\mu_{\pi^{0}}=0$. The scalar pion density is defined by $d n_{s \pi}=d n_{v \pi} /\left(2 E_{\pi}\right)$. We assume $-M_{\pi}<\mu_{\pi^{+}}<M_{\pi}$, so that a pion Bose condensate is not formed. Thermal properties of interacting pseudoscalar mesons $(\pi, K, \ldots)$ and nucleons are studied in Ref. [22].

The sum of the forward $\pi^{+} K, \pi^{0} K$, and $\pi^{-} K$ scattering amplitudes can be integrated over the pion momenta in the rest frame of the medium to obtain the $K$-meson self-energy operator: 


$$
\begin{aligned}
-\Sigma\left(p^{2}, E\right) & =\int A^{+}\left(s, 0, p^{2}\right)\left(d n_{s \pi^{+}}+d n_{s \pi^{0}}+d n_{s \pi^{-}}\right) \\
& +\int A^{-}\left(s, 0, p^{2}\right)\left(-d n_{s \pi^{+}}+d n_{s \pi^{-}}\right)
\end{aligned}
$$

Near threshold the amplitudes $A^{ \pm}\left(s, t, p^{2}\right)$ can be expanded up to $\left.\sim O\left(s-\left(M_{\pi}+M_{K}\right)^{2}\right)+O\left(p^{2}-M_{K}\right)^{2}\right)$, in which case the self-energy operator can be written as follows

$$
-\Sigma\left(p^{2}, E\right)=\left(p^{2}-M_{K}^{2}\right)\left(Z_{K}^{-1}-1\right)-\delta M_{K}^{2}-2 E V_{K} .
$$

This representation allows to identify $\delta M_{K}$ as a mass shift and $V_{K}$ as an external vector potential. The poles of the propagator appearing at $p^{2}-M_{K}^{2}-\Sigma\left(p^{2}, E\right)=0$ determine the in-medium kaon dispersion law

$$
E_{K}^{( \pm)}(\mathbf{p})= \pm \sqrt{\mathbf{p}^{2}+M_{K}^{2}+\delta M_{K}^{2}}+V_{K} .
$$

Using current algebra predictions for the threshold $\pi K$-scattering parameters [21], one gets

$$
\begin{aligned}
Z_{K^{ \pm}}^{[0]-1} & =1+\frac{n_{s \pi^{+}}+n_{s \pi^{0}}+n_{s \pi^{-}}}{2 F^{2}}, \\
\delta M_{K^{ \pm}}^{[0] 2} & =0, \\
V_{K^{ \pm}}^{[0]} & = \pm \frac{n_{v \pi^{+}}-n_{v \pi^{-}}}{4 F^{2}}
\end{aligned}
$$

where $F=92 \mathrm{MeV}$ is the pion decay constant. Ambiguities in the off-shell amplitudes due to different parameterizations of the pion field can be fixed at the tree level using the Adler self-consistency condition [21]. Eqs.(3) (7) are valid in tree approximation to first order in the pion density.

The results for $K^{0}$ and $\bar{K}^{0}$ can be obtained from isospin symmetry: $\delta M_{K^{ \pm}}^{[0]}=\delta M_{K^{0}}^{[0]}=\delta M_{\bar{K}^{0}}^{[0]}=0$ and $V_{K^{+}}^{[0]}=-V_{K^{-}}^{[0]}=-V_{K^{0}}^{[0]}=V_{\bar{K}^{0}}^{[0]}$. Since at RHIC energies $\sqrt{s_{N N}}=200 \mathrm{GeV}$ the charged pion number ratio $n_{\pi^{+}} / n_{\pi^{-}}$is very close to unity [23], the pion isovector density and the potential $V_{K}^{[0]}$ are negligible.

It is worthwhile to notice that the kaon dispersion law in nuclear matter has a similar structure with a negative mass shift $\delta M_{K}^{2}<0$ and a potential $V_{K^{+}}=-V_{K^{-}}>0$ $[1,2,5]$.

For pions the current algebra [21] gives $Z_{\pi^{ \pm}}^{-1}-1=$ $\left(n_{s \pi^{+}}+n_{s \pi^{-}}\right) / F^{2}, Z_{\pi^{0}}^{-1}-1=2 n_{s \pi^{0}} / F^{2}, \delta M_{\pi^{ \pm}}^{2}=$ $M_{\pi}^{2} n_{s \pi^{0}} / F^{2}, \delta M_{\pi^{0}}^{2}=M_{\pi}^{2}\left(n_{s \pi^{+}}+n_{s \pi^{-}}-n_{s \pi^{0}}\right) / F^{2}, V_{\pi^{ \pm}}=$ $\pm\left(n_{s \pi^{+}}-n_{s \pi^{-}}\right) / F^{2}$, and $V_{\pi^{0}}=0$. The pion mass correction at $\mu_{\pi}=0$ is in agreement with Ref. [24].

To lowest order ChPT isospin symmetric pion matter does not change the kaon dispersion law. The leading order effect appears at the one loop level. The effective mass of a quasiparticle has then to be determined from the relation $1 / m^{\text {eff }}=\partial^{2} \varepsilon(\mathbf{p}) / \partial|\mathbf{p}|^{2}$ at $\mathbf{p}=0$ where $\varepsilon(\mathbf{p})$ is the single particle energy. The vacuum ChPT corrections to the kaon self-energy are absorbed by $M^{2}$ and the vacuum renormalization constant of the kaon propagator and renormalize the scattering amplitudes (1). To lowest order in $\Sigma\left(p^{2}, E\right)=O\left(n_{v}\right)$ one obtains

$$
\varepsilon(\mathbf{p})=E_{\mathbf{p}}+\frac{\Sigma\left(M_{K}^{2}, E_{\mathbf{p}}\right)}{2 E_{\mathbf{p}}}
$$

with $E_{\mathbf{p}}=+\sqrt{\mathbf{p}^{2}+M_{K}^{2}}$. The mean-field potential

$$
V_{K}=\left.\frac{1}{2} \frac{\partial \Sigma\left(M_{K}^{2}, E\right)}{\partial E}\right|_{E=M_{K}}
$$

entering the dispersion law (4) determines the first-order shift of the self-energy operator from the kaon mass shell and leads automatically to the correct dispersion law to order $O\left(\mathbf{p}^{2} n_{v}\right)$. Given that the potential $V_{K}$ is known, the mass shift can be found from equation

$$
\delta M_{K}+V_{K}=\frac{\Sigma\left(M_{K}^{2}, M_{K}\right)}{2 M_{K}} .
$$

The values $\Sigma\left(M_{K}^{2}, M_{K}\right), \delta M_{K}$, and $V_{K}$ can be expressed in terms of the $s$ - and $p$-wave scattering lengths and the $s$-wave effective ranges. Using Eqs. (1) and (2), we obtain

$$
\Sigma\left(M_{K}^{2}, M_{K}\right)=-4 \pi n_{v} \frac{M_{\pi}+M_{K}}{M_{\pi}} a_{0}^{+}
$$

and

$$
V_{K}=-\frac{2 \pi n_{v}}{M_{\pi}+M_{K}}\left(a_{0}^{+}+2 M_{\pi} M_{K}\left(b_{0}^{+}+3 a_{1}^{+}\right)\right) .
$$

The corresponding mass shift can be found from Eq. (9). The self-energy operator (2) for kaons in isotopically symmetric pion matter has the same form as for antikaons due to $C$-parity. The values $\delta M_{K}$ and $V_{K}$ are also isoscalars, and so $\delta M_{K^{+}}=\delta M_{K^{0}}=\delta M_{K^{-}}=\delta M_{\bar{K}^{0}}$ and $V_{K+}=V_{K^{0}}=V_{K^{-}}=V_{\bar{K}^{0}}$, as distinct from the nuclear matter case $[1-3,5]$. Moreover, in isotopically symmetric pion matter $\Sigma\left(p^{2}, E\right)=\Sigma\left(p^{2},-E\right)$. This is a consequence of the crossing symmetry according to which $A^{ \pm}\left(s, 0, p^{2}\right)= \pm A^{ \pm}\left(u, 0, p^{2}\right)$ where $u=\left(p^{\prime}-p_{\pi}\right)^{2}$.

Current algebra predicts [21] $a_{0}^{+}=b_{0}^{+}+3 a_{1}^{+}=0$. The parameters $a_{0}^{+}, b_{0}^{+}$, and $a_{1}^{+}$entering into Eqs. (10) (11) receive in ChPT corrections to the order $p^{4}$. The calculations of Ref. [25] give $a_{0}^{+}=(0.023 \pm 0.012) / M_{\pi}$ and $b_{0}^{+}+3 a_{1}^{+}=(0.054 \pm 0.008) / M_{\pi}^{3}$. The representation (3) is valid when the amplitudes $A^{ \pm}\left(s, 0, p^{2}\right)$ are expanded near threshold up to $\left.O\left(s-\left(M_{\pi}+M_{K}\right)^{2}\right)+O\left(p^{2}-M_{K}\right)^{2}\right)$. The higher order terms in the expansion of the self-energy operator over the kaon momentum require the knowledge of the higher order threshold parameters. The next order terms, including higher partial waves $\ell \geq 2$, do not affect the results (10) - (11). Eqs. (9) - (11) provide thus a complete calculation of the kaon mass shift and the meanfield potential in ChPT to one loop, since the threshold parameters $a_{0}^{+}, b_{0}^{+}$, and $a_{1}^{+}$are evaluated to one loop. In symmetric pion matter $\delta M_{\pi} \sim O\left(1 / F^{2}\right)$ whereas $\delta M_{K} \sim$ 
$O\left(1 / F^{4}\right)$. ChPT off-shell ambiguities beyond the tree level have been investigated e.g. in $[24,26]$ from where one may conclude that physical observables are free from off-shell ambiguities at least up to the one loop level. To lowest order in the pion density, Eq.(2) and Eqs.(8) - (11) are thus generaly valid.

According to ChPT the pseudoscalar meson masses increase with temperature as expected for collective modes. A similar effect exists in the NJL model [12]. With increasing temperature, the constituent quark masses decrease, whereas the occupation numbers increase, pushing the $K$ mass up and the $\phi$ mass down. A decrease of the $\phi$-meson mass was also predicted by Asakawa and Ko [27].

Eqs. (10) - (11) are valid at $T \lesssim M_{\pi}$. The chemical freeze-out temperature at RHIC $T \sim 170 \mathrm{MeV}$ [23] is high, and so we should use a more phenomenological approach. We rewrite the $s$-wave parts of the amplitudes $A^{I}(s, t)$ in terms of phase shifts $\delta_{0}^{I}\left(p^{*}\right)=\left(a_{0}^{I} p^{*}+\left(b_{0}^{I}+\right.\right.$ $\left.\left.\frac{2}{3} a_{0}^{I 3}\right) p^{* 3}\right) \exp \left(-C_{I} \cdot \Phi_{2}^{4}\right)$, where $\Phi_{2}=\Phi_{2}\left(\sqrt{s}, M_{\pi}, M_{K}\right)=$ $\pi p^{*} / \sqrt{s}$ is the invariant $\pi K$ phase space, $C_{1 / 2}=0.75$ and $C_{3 / 2}=0.2$. The behavior of the $p$-wave is assumed to fixed by the $a_{1}^{I}$ scattering length and the resonance $K^{*}$. We thus make in the amplitudes substitutions

$$
\begin{aligned}
& a_{0}^{I}+b_{0}^{I} p^{* 2} \rightarrow e^{i \delta_{0}^{I}\left(p^{*}\right)} \sin \delta_{0}^{I}\left(p^{*}\right) / p^{*} \\
& a_{1}^{1 / 2} \rightarrow a_{1}^{1 / 2} \frac{\left|\left(M_{\pi}+M_{K}\right)^{2}-M_{K^{*}}^{2}+i M_{K^{*}} \Gamma_{K^{*}}\right|}{s-M_{K^{*}}^{2}+i M_{K^{*}} \Gamma_{K^{*}}} .
\end{aligned}
$$

The value $a_{1}^{3 / 2}$ is small and not modified. The amplitudes (12) and (13) satisfy unitarity. The experimental $\pi K$ scattering phases are then well reproduced, the low-temperature limit matches smoothly with one-loop ChPT. The amplitudes $A^{I}(s, t)$ are expressed in terms of the amplitudes $A^{ \pm}\left(s, t, p^{2}\right)$ as follows: $\left.\left.A^{1 / 2}(s, t)=A^{+}\left(s, t, M_{K}\right)^{2}\right)+2 A^{-}\left(s, t, M_{K}\right)^{2}\right)$ and $\left.\left.A^{3 / 2}(s, t)=A^{+}\left(s, t, M_{K}\right)^{2}\right)-A^{-}\left(s, t, M_{K}\right)^{2}\right)$.

The kaon self-energy at threshold, the mass shift and the mean-field potential are shown as a function of temperature in Fig. 1. Eq.(2) represents the self-energy in leading order in density which is the basic equation for the optical potential model [28]. The region of its validity is restricted to small wavelengths as compared to the length of the mean free path. This condition, $\lambda \sim 1 / p^{*} \lesssim 1 /\left(n_{v} \sigma^{t o t}\right)$, is equivalent to $m_{K} \Gamma_{K}^{*} / p^{* 2} \lesssim 1$. A simple estimate at $T=170 \mathrm{MeV}$ gives for the left hand side 0.08 , i.e. the criterion for the validity of Eq.(2) is satisfied.

At $T=170 \mathrm{MeV}$, we obtain $\delta M_{K}=-33 \mathrm{MeV}$ and $V_{K}=21 \mathrm{MeV}$. Thus the positive mass shift at low temperatures where $\mathrm{ChPT}$ is applicable becomes negative with increasing temperature. The value of $\Re \Sigma\left(M_{K}^{2}, M_{K}\right) /\left(2 M_{K}\right)$ remains relatively small up to $T=$ $200 \mathrm{MeV}$. The analogy with the Walecka model for nucleons is remarkable: The kaon mass shift at high temperatures is large and negative, the mean-field potential is large and positive, their sum is relatively small and negative. The kaons are therefore bound in pion matter similar to nucleons in nuclear matter. The mean-field potential is, however, $C$-even, as distinct from the case of nucleons and kaons in nuclear matter $[1,2,5]$ and the case of kaons in asymmetric pion matter, as discussed above (see Eq. (7)).

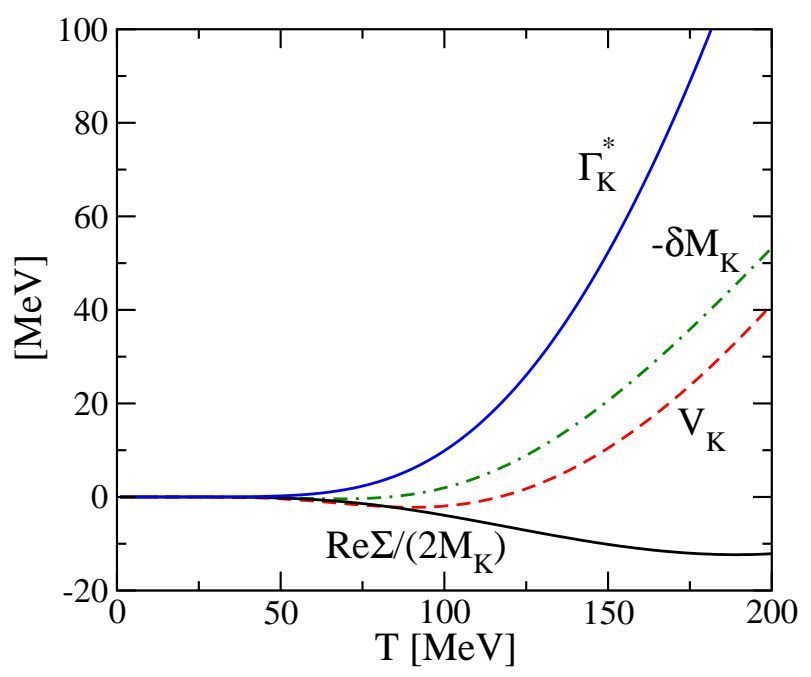

FIG. 1. Self-energy $\Re \Sigma\left(M_{K}^{2}, M_{K}\right) /\left(2 M_{K}\right)$ of kaons at rest, mass shift $-\delta M_{K}$, mean-field potential $V_{K}$, and kaon collision width $\Gamma_{K}^{*}$ versus temperature $T$ in isotopically symmetric pion matter.

The $\phi$-meson collision broadening has been discussed in Refs. $[27,29,30]$. The physics behind this effect is the same as in the collision broadening of the atomic spectral lines in hot and dense gases, discussed by Weisskopf [31] in the early 1930's. The $\phi \rightarrow K \bar{K}$ decay might be suppressed due to a increasing kaon mass and/or a decreasing $\phi$-meson mass. Collisions of the $\phi$-meson and the kaons keep, however, the $\phi \rightarrow K \bar{K}$ decay channel open even at $M_{\phi}<2 M_{K}$ and result in an increase of the total $\phi$ width, both at $M_{\phi}<2 M_{K}$ and $M_{\phi}>2 M_{K}$.

The kaon collision width can be found from equation

$$
\begin{aligned}
\Gamma_{K}^{*} & =\frac{1}{6 M_{K}(2 \pi)^{2}} \sum_{\ell} \int\left(\left|A_{\ell}^{1 / 2}(s)\right|^{2}+2\left|A_{\ell}^{3 / 2}(s)\right|^{2}\right) \\
& \times d n_{s} \Phi_{2}\left(\sqrt{s}, M_{\pi}, M_{K}\right)
\end{aligned}
$$

where $A_{\ell}^{I}(s)$ are the on-shell partial wave projections of the $\pi K$ amplitudes with total isospin $I=1 / 2$ and $3 / 2$. The $K^{*}$ increases the width by $\sim 40 \mathrm{MeV}$. As a result, we get $\Gamma_{K}^{*}=81 \mathrm{MeV}$ at $T=170 \mathrm{MeV}$. The kaon collision width as a function of temperature is shown in Fig. 1.

The $\phi$-meson in-medium width can be written as $\Gamma_{\phi \rightarrow K \bar{K}}^{\text {med }}=\eta \Gamma_{\phi \rightarrow K \bar{K}}^{\mathrm{vac}}$ where

$$
\begin{aligned}
\eta= & \frac{1}{\pi^{2}} \int \frac{p^{* 3}\left(M_{\phi}^{*}, m_{1}^{*}, m_{2}^{*}\right)}{p^{* 3}\left(M_{\phi}, M_{K}, M_{K}\right)} \frac{M_{K}^{*} \Gamma_{K}^{*} d m_{1}^{* 2}}{\left(m_{1}^{* 2}-M_{K}^{* 2}\right)^{2}+\left(M_{K}^{*} \Gamma_{K}^{*}\right)^{2}} \\
& \times \frac{M_{K}^{*} \Gamma_{K}^{*} d m_{2}^{* 2}}{\left(m_{2}^{* 2}-M_{K}^{* 2}\right)^{2}+\left(M_{K}^{*} \Gamma_{K}^{*}\right)^{2}} .
\end{aligned}
$$


We set $M_{\phi}^{*}=M_{\phi}-2 V_{K}$ and $M_{K}^{*}=M_{K}+\delta M_{K}$. Any modification of the real part of the kaon self-energy does not significantly alter the in-medium $\phi$-meson decay rate as long as $\Re \Sigma\left(M_{K}^{2}, M_{K}\right)<<\varsigma \Sigma\left(M_{K}^{2}, M_{K}\right)=M_{K} \Gamma_{K}^{*}$. As can be seen from Fig. 1 this is the present case. The enhancement factor $\eta$ as a function of temperature is shown in Fig. 2. At $T=170 \mathrm{MeV}$, we get $\eta \sim 3$ and $\Gamma_{\phi}^{\text {med }} \sim 12 \mathrm{MeV}$. The in-medium $\phi$-meson width increases, since the Breit-Wigner distribution allows an effective reduction of the kaon masses and, as a consequence, an effective increase of the available phase space.

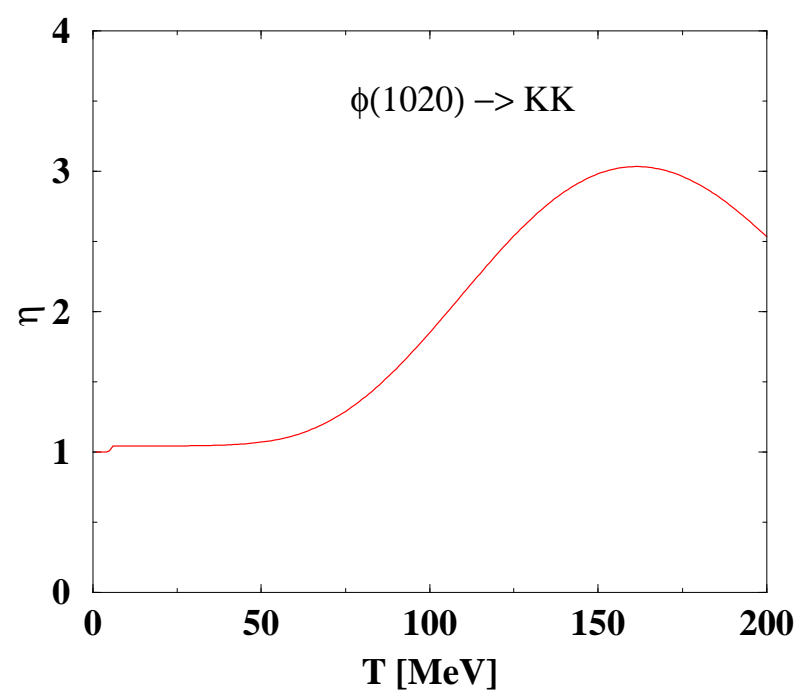

FIG. 2. Enhancement factor $\eta$ of the $\phi \rightarrow K \bar{K}$ decay versus temperature $T$ in isotopically symmetric pion matter.

Dileptons from $\phi$ decays leave the pion matter essentially undistorted by final-state interactions, whereas the secondary kaons rescatter and can contribute to the experimental background. This can result in an increase of the apparent dilepton branching, as one sees from the following arguments: Let us assume that $\phi$-mesons leave the reaction at time $\tau$ after their creation and that kaons originating from in-medium $\phi$-mesons do not rescatter. The $\phi \rightarrow K \bar{K}$ decays generate nonrelativistic $K$-mesons, in the $\phi$-meson c. m. frame. Hence, secondary kaons move with the same velocity as $\phi$-mesons and leave the reaction zone without rescattering with probability

$$
w \sim \int_{0}^{\tau}\left(e^{-\Gamma_{K}^{*}(\tau-t)}\right)^{2} e^{-\Gamma_{\phi}^{*} t} \Gamma_{\phi} d t .
$$

The first term is the probability for two kaons to escape from the reaction zone without rescattering. The second term is the survival probability of the $\phi$-meson at time $t$. The last term $\Gamma_{\phi} d t$ is the probability to decay into the kaon pair during $d t$. Notice that $\Gamma_{\phi}^{*} d t$ has the meaning of a decay probability into the kaons which rescatter with pions. We select pairs with invariant masses of the $\phi$ meson which suffered no rescatterings. The number of the $\phi$-mesons observed in the two-kaon channel equals therefore

$$
N_{K \bar{K}} \sim e^{-\Gamma_{\phi}^{*} \tau}+\frac{\Gamma_{\phi}}{2 \Gamma_{K}^{*}-\Gamma_{\phi}^{*}}\left(e^{-\Gamma_{\phi}^{*} \tau}-e^{-2 \Gamma_{K}^{*} \tau}\right)
$$

The first term arises due to the vacuum decays, whereas the second term is given by Eq. (15). The number of the $\phi$-mesons observed in the dilepton channel equals

$$
N_{e^{+} e^{-}} \sim e^{-\Gamma_{\phi}^{*} \tau} B+\left(1-e^{-\Gamma_{\phi}^{*} \tau}\right) B^{*}
$$

where $B$ and $B^{*}$ are the vacuum and in-medium dilepton branchings of the $\phi$-mesons. We assume that the dilepton channel is not modified, $B^{*} / B=\Gamma_{\phi} / \Gamma_{\phi}^{*}$. The apparent dilepton branching becomes

$$
B^{\text {app }}=B \frac{1+\left(e^{\Gamma_{\phi}^{*} \tau}-1\right) \Gamma_{\phi} / \Gamma_{\phi}^{*}}{1+\frac{\Gamma_{\phi}}{2 \Gamma_{K}^{*}-\Gamma_{\phi}^{*}}\left(1-e^{-\left(2 \Gamma_{K}^{*}-\Gamma_{\phi}^{*}\right) \tau}\right)} .
$$

According to transport calculations $e^{-\tau \Gamma_{\phi}} \sim 1 / 2$ at RHIC energies [32]. In such a case, varying the temperature $T=120 \div 170$ between the commonly accepted values for thermal and chemical freeze-out, we get $B^{\text {app }} / B \sim 2 \div 3$ which is in qualitative agreement with the observations from NA50, NA49, and PHENIX collaborations who report an increased apparent dilepton branching. Future data from RHIC and additional studies incorporating refined fireball dynamics as well as in-medium meson modifications might help to completely solve the $\phi$ puzzle and bring more insight in the meson propagation in a dense and hot medium.

\section{ACKNOWLEDGMENTS}

This work is supported by GSI (Darmstadt) under the contract TÜFÄST, by RFBR grant No. 03-02-04004, DFG grant No. 436 RUS 113/721/0-1, and by Federal Program of the Russian Ministry of Industry, Science and Technology No. 40.052.1.1.1112.

[1] D.B. Kaplan and A.E. Nelson, Phys. Lett. B175 (1986)57.

[2] G.E. Brown and M. Rho, Phys. Reports 269 (1996) 333.

[3] T. Waas, N. Kaiser, and W. Weise, Phys. Lett. B365 (1996) 12; M. Lutz, Phys. Lett. B426 (1998) 12.

[4] Y. Shin, et al., KaoS Collaboration, Phys. Rev. Lett. 81 (1998) 1576;

F. Laue, et al., KaoS Collaboration, Phys. Rev. Lett. 82 (1999)1640;

P. Crochet et al., FOPI Collaboration, Phys. Lett. B486 (2000) 6;

C. Sturm et al., KaoS Collaboration, Phys. Rev. Lett. 86 (2001) 39.

[5] G.Q. Li et al., Phys. Rev. Lett. 74 (1995) 235; Nucl. Phys. A625 (1997) 327; Nucl. Phys. A636 (1998) 4887. 
[6] Z.S. Wang et al., Phys. Rev. Lett. 79 (1997) 4096;

C. Fuchs et al., Phys. Lett. B434 (1998) 245; Phys. Rev. Lett. 86 (2001) 1794.

[7] M. Nekipelov et al., ANKE-Collaboration, Phys. Lett. B540 (2002) 207.

[8] L.V. Bravina, Amand Faessler, C. Fuchs, E.E. Zabrodin, Z.-D. Lu, Phys. Rev. C 66 (2002) 014906;

K. Adcox et al., PHENIX Collaboration, Phys. Rev. Lett. 86 (2001) 3500;

B.B. Back et al., PHOBOS Collaboration, Phys. Rev. C65 (2002) 031901(R).

[9] P. Koch, B. Müller, and J. Rafelski, Phys. Rep. 142 (1986) 1.

[10] A. Shor, Phys. Rev. Lett. 54 (1985) 1122.

[11] D. Lissauer and E.V. Shuryak, Phys. Lett. B253 (1991) 15.

[12] J.-P. Blaizot and R. M. Galain, Phys. Lett. B271 (1991) 32.

[13] N. Willis [for NA50 Collaboration], Nucl. Phys. A661 (1999) 534c.

[14] C. Hoehne [for NA49 Collaboration], Nucl. Phys. A661 (1999) 485c.

[15] S.C. Johnson, B.V. Jacak, and A. Drees, Eur. Phys. J. C18 (2001) 645.

[16] S. Soff et al., J. Phys. G27 (2001) 449;

S. Pal, C.M. Ko, and Z.-W. Lin, Nucl. Phys. A707 (2002) 525 .

[17] J.L. Nagle et al., PHENIX Collaboration, nuclex/0209015.

[18] A. Dobado, J.R. Peláez, Phys. Rev. D59 (1999) 034004.

[19] J.R. Peláez, Phys. Rev. D66 (2002) 096007.

[20] A. G. Nicola and J. R. Peláez, Phys. Rev. D65 (2002) 054009 .

[21] J.A. Cronin, Phys. Rev. 161 (1967) 1483; R.W. Griffith, 176 (1968) 1705.

[22] R. Venugopalan and M. Prakash, Nucl. Phys. A546 (1992) 718.

[23] P. Braun-Munzinger, D. Magestro and K. Redlich, Phys. Lett. B518 (2001) 41.

[24] J. Gasser and H. Leutwyler, Phys. Lett. B184 (1987) 83; Ann. Phys. 158 (1984) 142.

[25] V. Bernard, N. Kaiser and Ulf-G. Meeissner, Phys. Rev. D43 (1991) R2757; Nucl. Phys. B357 (1991) 129.

[26] S. Scherer, hep-ph/0210398.

[27] M. Asakawa and C.M. Ko, Phys. Lett. B322 (1994) 33; Phys. Rev. C50 (1994) 3064.

[28] L. D. Landau and E. M. Lifshitz, Quantum Mechanics. Nonrelativistic Theory. 4 edn. (Moscow, Nauka, 1989).

[29] D. Seibert and C. Gale, Phys. Rev. C52 (1995) 490.

[30] L. Alvarez-Ruso and V. Koch, Phys. Rev. C65 (2002) 054901.

[31] V. Weisskopf, Physikalische Zeitschrift 34 (1933) 1.

[32] L. Bravina et al., Nucl. Phys. A715 (2003) 665c. 